Proyecciones Journal of Mathematics

Vol. 36, $\mathrm{N}^{\circ}$ 1, pp. 117-130, March 2017.

Universidad Católica del Norte

Antofagasta - Chile

\title{
Some $I$-convergent triple sequence spaces defined by a sequence of modulus function
}

\author{
Bimal Chandra Das \\ Tripura University, India \\ Received : September 2016. Accepted : October 2016
}

\begin{abstract}
In this article we introduce the notion of I-convergent triple sequence spaces $c_{0 I}^{3}(F), c_{I}^{3}(F), l_{\infty I}^{3}(F), m_{I}^{3}(F)$ and $m_{0 I}^{3}(F)$ defined by a sequence of modulii $F=\left(f_{p q r}\right)$ and study some of their algebraic and topological properties like solidity, symmetricity, convergence free etc. We also prove some inclusion relation involving these sequence spaces.
\end{abstract}

Key Words : Triple sequence, Modulus function, I- convergence, Ideal, filter

AMS Classification : 40A05, 40A35, 40C05, 46A45. 


\section{Introduction}

Throughout the article a triple sequence $x$ is denoted by $\left(x_{p q r}\right)$ i.e. a triple infinite array of real or complex numbers $x_{p q r}, p, q, r \in N$. Throughout the article $c_{0 I}^{3}(F), c_{I}^{3}(F), l_{\infty I}^{3}(F), m_{I}^{3}(F)$ and $m_{0 I}^{3}(F)$ denote the triple sequence spaces of $I$ - null in Pringshiems sense , $I$ - convergent in Pringshiems sense, $I$ - bounded in Pringshiems sense, bounded $I$-convergence and bounded $I$ null in Pringshiems sense respectively. Throughout N, R and C denote the set of natural, real and complex numbers respectively.

Let $w^{3}$ denote the set of all triple sequences of real or complex numbers. Then the classes of triple sequences $c_{0}^{3}, c^{3}, l_{\infty}^{3}, c^{3 R}$ and $c^{3 B}$ denote the triple sequence spaces which are convergent to zero in Pringsheim's sense, convergent in Pringsheim's sense, bounded in Pringsheim's sense, regularly convergent, bounded and convergent respectively.

The notion of statistical convergence was first introduced by Fast [11] and Schoenberg [13] independently. Kumar [19] generalized this concept in probabilistic normed space. Khan and Khan ([20],[21]) introduced the $I$ -convergence double sequence spaces by modulus function and sequence of modulii respectively. The notion of statistical convergent double sequence was introduced by Tripathy [4] and extended this concept in $I$-convergent double sequence. Tripathy and Sen [5] studied for double sequence space associated with multiplier sequences.

The notion of I-convergent is a generalization of the statistical convergence. $I$-convergence of real sequence was introduced at the initial stage by Kostyrko, Salat and Wilczynski [14]. Later on it was studied by Salat, Tripathy and Ziman [18] and many other researchers. Tripathy and Goswami [6] extended this concept in probabilistic normed space using triple difference sequences of real numbers.

At the initial stage the different types of notions of triple sequences was introduced and investigated by Sahiner, Gurdal and Duden [3]. Dutta, Esi and Tripathy [1] generalizes this concept by using Orlicz function. Savas and Patterson [10] introduced double sequence spaces defined by a modulus function. Sahiner and Tripathy [2] studied $I$-related properties in triple sequence spaces and showed some interesting results. Recently Tripathy and Goswami ([7],[8]) studied vector valued multiple sequences by using Orlicz 
function and multiple sequences in probabilistic normed spaces respectively. Debnath, Sharma and Das [16] and Debnath and Das [15] generalized these concepts by using the difference operator.

\section{Definitions and Preliminaries}

Definition 2.1. Let $X \neq \emptyset$. A class $I$ of subsets of $X$ is said to be an ideal in $X$ provided:

(i) $\emptyset \in I$.

(ii) $A, B \in I$ implies $A \cup B \in I$.

(iii) $A \in I, B \subset A$ implies $B \in I$.

I is called a non-trivial ideal if $X \notin I$.

Definition 2.2. Let $X \neq \emptyset$. A non empty class $F$ of subsets of $X$ is said to be a filter in $X$ provided:

(i) $\emptyset \notin F$.

(ii) $A, B \in F$ implies $A \cap B \in F$.

(iii) $A \in F, A \subset B$ implies $B \in F$.

If $I$ is a nontrivial ideal in $X, X \neq \emptyset$ then the class.

$F(I)=\{M \subset X:(\exists A \in I)(M=X \backslash A)\}$

is a filter on $X$, called the filter associated with $I$.

Definition 2.3. A nontrivial ideal $I$ in $X$ is called admissible if $\{x\} \in I$ for each $x \in X$.

Definition 2.4. A non-trivial ideal $I$ is maximal if there cannot exist any non-trivial ideal $J \neq I$ containing $I$ as a subset. 
Definition 2.5. A triple sequence $\left(x_{p q r}\right)$ is said to be convergent to $L$ in Pringsheim's sense if for every $\varepsilon>0$, there exists $n_{0} \in N$ such that

$$
\left|x_{p q r}-L\right|<\varepsilon, \text { whenever } p \geq n_{0}, q \geq n_{0}, r \geq n_{0},
$$

and we write $\lim _{p, q, r \rightarrow \infty} x_{p q r}=L$.

Note: A triple sequence convergent in Pringsheim's sense is not necessarily bounded [3]. This is clear from the following example.

Example 2.1. Consider the sequence $\left(x_{p q r}\right)$ defined by

$$
x_{p q r}=\left\{\begin{array}{l}
p \quad \text { for all } p \in N, \quad q=1=r \\
\frac{1}{p+q+r} \quad \text { otherwise }
\end{array}\right.
$$

Then $x_{p q r} \rightarrow 0$ in Pringsheim's sense but is unbounded.

Definition 2.6. A triple sequence $\left(x_{p q r}\right)$ is said to be $I$-convergence to a number L if for every $\left.\varepsilon>0,\left\{(p, q, r) \in N \times N \times N:\left|x_{p q r}-L\right|\right) \geq \varepsilon\right\} \in I$. in this case we write $I-\lim x_{p q r}=L$.

Definition 2.7. A triple sequence $\left(x_{p q r}\right)$ is said to be $I$-null if $L=0$. In this case we write $I-\lim x_{p q r}=0$.

Definition 2.8. A triple sequence $\left(x_{p q r}\right)$ is said to be Cauchy sequence if for every $\varepsilon>0$, there exists $n_{0} \in N$ such that $\left|x_{p q r}-x_{l m n}\right|<\varepsilon$, whenever $p \geq l \geq n_{0}, q \geq m \geq n_{0}, r \geq n \geq n_{0}$.

Definition 2.9. A triple sequence $\left(x_{p q r}\right)$ is said to be $I$-Cauchy if for every $\varepsilon>0$, there exists $l=l_{0}, m=m_{0}$ and $n=n_{0}$ such that $\{(p, q, r) \in$ $\left.N \times N \times N:\left|x_{p q r}-x_{l m n}\right| \geq \varepsilon\right\} \in I$.

Definition 2.10. A triple sequence $\left(x_{p q r}\right)$ is said to be $I$-bounded if there exists $M>0$ such that $\left\{(p, q, r) \in N \times N \times N:\left|x_{p q r}\right|>M\right\} \in I$.

These classes are all linear spaces. 
It is obvious that $c_{0}^{3} \subset c^{3}, c^{3 R} \subset c^{3 B} \subset l_{\infty}^{3}$ and the inclusion are strict.

We state the following result without proof.

Theorem 2.1. The spaces $c_{0}^{3}, c^{3}, l_{\infty}^{3}, c^{3 R}$ and $c^{3 B}$ are complete normed linear spaces with the norm.

$$
\|x\|=\sup _{p, q, r}\left|x_{p q r}\right|<\infty
$$

Definition 2.11. A triple sequence space $\mathrm{E}$ is said to be solid if $\left(\alpha_{p q r} x_{p q r}\right) \in$ $E$ whenever $\left(x_{p q r}\right) \in E$ and for all sequences $\left(\alpha_{p q r}\right)$ of scalars with $\left|\alpha_{p q r}\right| \leq$ 1 , for all $p, q, r \in N$.

Definition 2.12. A triple sequence space $E$ is said to be symmetric if $\left(x_{p q r}\right) \in E$ implies $\left(x_{\pi(p, q, r)}\right) \in E$, where $\pi$ is a permutation of $N \times N \times N$.

Definition 2.13. A triple sequence space $\mathrm{E}$ is said to be monotone if it contains the canonical pre-images of all its step spaces.

Remark: A sequence space is solid implies that it is monotone.

Definition 2.14. Let $K=\left\{\left(i_{p}, j_{q}, k_{r}\right):(p, q, r) \in N \times N \times N: i_{1}<i_{2}<\right.$ $i_{3}<\ldots \ldots . ., j_{1}<j_{2}<j_{3}<\ldots \ldots$ and $\left.k_{1}<k_{2}<k_{3}<\ldots \ldots.\right\} \subseteq N \times N \times N$ and $E$ is the triple sequence space. A $K$-stepspace of $E$ is a sequence space $\lambda_{K}^{E}=\left\{\left(\alpha_{p q r} x_{p q r}\right):\left(x_{p q r}\right) \in E\right\}$.

Definition 2.15. A triple sequence space $\mathrm{E}$ is said to be convergence free if $\left(y_{p q r}\right) \in E$, whenever $\left(x_{p q r}\right) \in E$ and $x_{p q r}=0$ implies $y_{p q r}=0$.

Definition 2.16. A triple sequence space $\mathrm{E}$ is said to be sequence algebra if $\left(x_{p q r} \star y_{p q r}\right) \in E$, whenever $\left(x_{p q r}\right) \in E$ and $y_{p q r} \in E$.

Lemma 2.1. Let $I \subset 2^{N}$ and $M \subseteq N$. If $M \notin I$, then $M \cap N \notin I$

Definition 2.17. A function $f:[0, \infty) \rightarrow[0, \infty)$ is called a modulus function if it satisfies the following four conditions [12]: 
(1) $f(x)=0$ if and only if $x=0$

(2) $f(x+y) \leq f(x)+f(y)$ for all $x \geq 0$ and $y \geq 0$

(3) $f$ is increasing.

(4) $f$ is continuous from the right at 0 .

Since $|f(x)-f(y)| \leq f(|x-y|)$, it follows from condition (4) that $f$ is continuous on $[0, \infty)$. Furthermore, from condition (2) we have $f(n x) \leq$ $n f(x)$, for all $n \in N$, and so

$$
f(x)=f\left(n x\left(\frac{1}{n}\right)\right) \leq n f\left(\frac{x}{n}\right), \text { Hence } \frac{1}{n} f(x) \leq f\left(\frac{x}{n}\right)
$$

for all $n \in N$. A modulus function may not be bounded. For example, $f(x)=x^{p}$, for $0<p \leq 1$ is unbounded, but $f(x)=\frac{x}{1+x}$ is bounded.

Now we introduce the following triple sequence spaces, where $F=\left(f_{p q r}\right)$ is the sequence of modulii :

$$
\begin{aligned}
& c_{0 I}^{3}(F)=\left\{x \in w^{3}: I-\lim f_{p q r}\left(\left|x_{p q r}\right|\right)=0\right\} \in I \\
& c_{I}^{3}(F)=\left\{x \in w^{3}: I-\lim f_{p q r}\left(\left|x_{p q r}-L\right|\right)=0, \text { for some } L\right\} \in \mathrm{I} \\
& l_{\infty I}^{3}(F)=\left\{x \in w^{3}: \quad{ }_{p, q}, r \in N\right. \\
& m_{I}^{3}(F)=c_{I}^{3}(F) \cap l_{\infty I}^{3}(F) \\
& m_{0 I}^{3}(F)=c_{0 I}^{3}(F) \cap l_{\infty I}^{3}(F)
\end{aligned}
$$

Where $c_{0 I}^{3}(F), c_{I}^{3}(F), l_{\infty I}^{3}(F), m_{I}^{3}(F)$ and $m_{0 I}^{3}(F)$ are the triple sequence spaces $I$ - null in Pringshiems sense , $I$ - convergent in Pringshiems sense, $I$ - bounded in Pringshiems sense, bounded $I$-convergence and bounded $I$ - null in Pringshiems sense respectively.

The main aim of our study is to extend the concept of ideal convergence from double sequences to triple sequences defined by modulli function and establish some important results. 


\section{Main Results}

Theorem 3.1. The triple sequence spaces $c_{0 I}^{3}(F), c_{I}^{3}(F), l_{\infty I}^{3}(F), m_{I}^{3}(F)$ and $m_{0 I}^{3}(F)$ all are linear for the sequence of modulii $F=\left(f_{p q r}\right)$.

Proof. We shall prove it for the sequence space $c_{I}^{3}(F)$, for the other spaces, it can be established similarly.

We assume that $\left(x_{p q r}\right),\left(y_{p q r}\right) \in c_{I}^{3}(F)$ and $\alpha, \beta$ be two scalars such that $|\alpha| \leq 1$ and $|\beta| \leq 1$

Then

$I-\lim f_{p q r}\left(\left|x_{p q r}-L_{1}\right|\right)=0$, for some $L_{1} \in C$

$I-\lim f_{p q r}\left(\left|y_{p q r}-L_{2}\right|\right)=0$, for some $L_{2} \in C$

Now for a given $\varepsilon>0$ we can write

$$
\begin{aligned}
& A_{1}=\left\{(p, q, r) \in N \times N \times N: f_{p q r}\left(\left|x_{p q r}-L_{1}\right|\right)>\frac{\varepsilon}{2}\right\} \in I \\
& A_{2}=\left\{(p, q, r) \in N \times N \times N: f_{p q r}\left(\left|y_{p q r}-L_{2}\right|\right)>\frac{\varepsilon}{2}\right\} \in I
\end{aligned}
$$

Since $f_{p q r}$ is a modulus function, so it is non-decreasing and convex, hence we get

$$
\begin{gathered}
f_{p q r}\left(\left|\left(\alpha x_{p q r}+\beta y_{p q r}\right)-\left(\alpha L_{1}+\beta L_{2}\right)\right|\right)=f_{p q r}\left(\mid\left(\alpha x_{p q r}-\alpha L_{1}\right)\right. \\
\left.+\left(\beta y_{p q r}-\beta L_{2}\right) \mid\right) \\
\leq f_{p q r}\left(|\alpha|\left|x_{p q r}-L_{1}\right|\right)+f_{p q r}\left(|\beta|\left|y_{p q r}-L_{2}\right|\right)=|\alpha| f_{p q r}\left(\left|x_{p q r}-L_{1}\right|\right) \\
+|\beta| f_{p q r}\left(\left|y_{p q r}-L_{2}\right|\right) \\
\left.\leq f_{p q r}\left(\left|x_{p q r}-L_{1}\right|\right)+f_{p q r}\left(\left|y_{p q r}-L_{2}\right|\right)\right)
\end{gathered}
$$


From (2.1) and (2.2) we can write

$\left\{p, q, r \in N \times N \times N: f_{p q r}\left(\left|\left(\alpha x_{p q r}+\beta y_{p q r}\right)-\left(\alpha L_{1}+\beta L_{2}\right)\right|\right)>\varepsilon\right\} \subset A_{1} \cup A_{2}$

Thus $\alpha x_{p q r}+\beta y_{p q r} \in c_{I}^{3}(F)$

This completes the proof.

Theorem 3.2. A triple sequence of real or complex numbers $x=\left(x_{p q r}\right) \in m_{I}^{3}(F)$ is I convergence iff for every $\varepsilon>0$ there exist $I_{\varepsilon}, J_{\varepsilon}, K_{\varepsilon} \in N$ such that

$$
\left.\left\{(p, q, r) \in N \times N \times N: f_{p q r}\left(\left|x_{p q r}-x_{I_{\varepsilon}, J_{\varepsilon}, K_{\varepsilon}}\right|\right) \leq \varepsilon\right\} \in m_{I}^{3}(F)\right\}
$$

Proof. Let $L=I-\lim x$. Then we have

$$
\begin{aligned}
\quad A_{\varepsilon}=\left\{(p, q, r) \in N \times N \times N: f_{p q r}\left(\mid\left(x_{p q r}-L\right) \leq \frac{\varepsilon}{2}\right\} \in m_{I}^{3}(F)\right\} \text { for all, } \\
\varepsilon>0
\end{aligned}
$$

Next fix $I_{\varepsilon}, J_{\varepsilon}, K_{\varepsilon} \in A_{\varepsilon}$ then we have

$$
\left|x_{p q r}-x_{I_{\varepsilon}, J_{\varepsilon}, K_{\varepsilon}}\right| \leq\left|x_{p q r}-L\right|+\left|L-x_{I_{\varepsilon}, J_{\varepsilon}, K_{\varepsilon}}\right| \leq \frac{\varepsilon}{2}+\frac{\varepsilon}{2}=\varepsilon \text { for all, } p, q, r \in A_{\varepsilon}
$$

Thus $\left.\left\{(p, q, r) \in N \times N \times N: f_{p q r}\left(\left|x_{p q r}-x_{I_{\varepsilon}, J_{\varepsilon}, K_{\varepsilon}}\right|\right) \leq \varepsilon\right\} \in m_{I}^{3}(f)\right\}$

Conversely, suppose that

$$
\left.\left\{(p, q, r) \in N \times N \times N: f_{p q r}\left(\left|x_{p q r}-x_{I_{\varepsilon}, J_{\varepsilon}, K_{\varepsilon}}\right|\right) \leq \varepsilon\right\} \in m_{I}^{3}(F)\right\}
$$

we get $\left.\left\{(p, q, r) \in N \times N \times N: f_{p q r}\left(\left|x_{p q r}-x_{I_{\varepsilon}, J_{\varepsilon}, K_{\varepsilon}}\right|\right) \leq \varepsilon\right\} \in m_{I}^{3}(F)\right\}$ for all, $\varepsilon>0$

Then we can find the set

$$
\underset{m_{I}^{3}(F)}{B_{\varepsilon}}=\left\{(p, q, r) \in N \times N \times N: x_{p q r} \in\left[x_{I_{\varepsilon}, J_{\varepsilon}, K_{\varepsilon}}-\varepsilon, x_{I_{\varepsilon}, J_{\varepsilon}, K_{\varepsilon}}+\varepsilon\right]\right\} \in
$$

given $\varepsilon>0$. 
Consider $N_{\varepsilon}=\left[x_{I_{\varepsilon}, J_{\varepsilon}, K_{\varepsilon}}-\varepsilon, x_{I_{\varepsilon}, J_{\varepsilon}, K_{\varepsilon}}+\varepsilon\right]$

Now we have $B_{\varepsilon} \in m_{I}^{3}(F)$ as well as $B_{\frac{\varepsilon}{2}} \in m_{I}^{3}(F)$

Hence $B_{\varepsilon} \cap B_{\frac{\varepsilon}{2}} \in m_{I}^{3}(F)$ which implies $M_{\varepsilon} \cap M_{\frac{\varepsilon}{2}} \neq \emptyset$

Then $\left\{(p, q, r) \in N \times N \times N: x_{p q r} \in N\right\} \in m_{I}^{3}(F)$

which implies $\operatorname{diam} M \leq \operatorname{diam} M_{\varepsilon}$

where the diam of $M$ denotes the length of interval $N$.

In this way by principal of induction we found the sequence of closed intervals

$$
M_{\varepsilon}=I_{0} \supseteq I_{1} \supseteq I_{2} \supseteq \ldots \ldots \supseteq I_{s} \supseteq \ldots \ldots \ldots
$$

With the help of the property that $\operatorname{diam} I_{s} \leq \frac{1}{2} \operatorname{diam} I_{s-1}$, for $(s=1,2,3,4, \ldots \ldots \ldots .$.$) and \left\{(p, q, r) \in N \times N \times N: x_{p q r} \in I_{p q r}\right\} \in m_{I}^{3}(F)$, for $(p, q, r=1,2,3,4, \ldots \ldots \ldots \ldots)$

Then there exist a $\xi \in \cap I_{s}$ where $s \in N$ such that $\xi=I-\lim x$

So that $f_{p q r}(\xi)=I-\lim f_{p q r}(x)$ therefore $L=I-\lim f_{p q r}(x)$

Hence the proof is complete.

Theorem 3.3. The inclusions $c_{0 I}^{3}(F) \subset c_{I}^{3}(F) \subset l_{\infty I}^{3}(F)$ are strict, where $F$ is the function of modulii.

Proof. We consider $x_{p q r} \in c_{I}^{3}(F)$. Then there exist $L \in C$ such that

$I-\lim f_{p q r}\left(\left|x_{p q r}-L\right|\right)=0$, we get $f_{p q r}\left(\left|x_{p q r}\right|\right) \leq f_{p q r}\left(\left|x_{p q r}-L\right|\right)+$ $f_{p q r}(|L|)$

On taking supremum over $p, q$ and $r$ on both sides gives

$$
\left(x_{p q r}\right) \in l_{\infty I}^{3}(F)
$$


The inclusion $c_{0 I}^{3}(F) \subset c_{I}^{3}(F)$ is obvious

Hence the inclusion $c_{0 I}^{3}(F) \subset c_{I}^{3}(F) \subset l_{\infty I}^{3}(F)$ are strict.

Theorem 3.4. The triple sequence spaces $c_{0 I}^{3}(F)$ and $m_{0 I}^{3}(F)$ are solid.

Proof. We shall prove the result for $c_{0 I}^{3}(F)$ and the result for $m_{0 I}^{3}(F)$ can be established similarly .

Consider $x_{p q r} \in c_{0 I}^{3}(F)$ and then $I-\lim _{p q r} f_{p q r}\left(\left|x_{p q r}\right|\right)=0$

Now we consider a sequence of scalar $\left(\alpha_{p q r}\right)$ such that $\left|\alpha_{p q r}\right| \leq 1$, for all $p, q, r \in N$

Then we have,

$$
\begin{aligned}
& I-{ }_{p q r}^{\lim } f_{p q r}\left(\left|\alpha_{p q r} x_{p q r}\right|\right) \leq I-{ }_{p q r}^{\lim } f_{p q r}\left(\left|\alpha_{p q r}\right|\left|x_{p q r}\right|\right) \\
& =\left|\alpha_{p q r}\right| I-{ }_{p q r} f_{p q r}\left(\left|x_{p q r}\right|\right)=0
\end{aligned}
$$

Hence $I-{ }_{p q r}^{\lim } f_{p q r}\left(\left|\alpha_{p q r} x_{p q r}\right|\right)=0$ for all $p, q, r \in N$

This implies $\alpha_{p q r} x_{p q r} \in c_{0 I}^{3}(F)$

Therefor the space $c_{0 I}^{3}(F)$ is solid.

Hence the proof.

Theorem 3.5. The triple sequence spaces $c_{I}^{3}(F)$ and $m_{I}^{3}(F)$ are not monotonic in general.

Proof. We take a counter example to prove the result for $c_{I}^{3}(F)$. The other part can be proved similarly.

Let $I=I_{\delta}$ and $f(x)=x$, for all $x \in[0, \infty)$

Consider the $K$-step space $X_{K}(F)$ of $X$ defined as follows: 
Now we assume $x_{p q r} \in X$ and $y_{p q r} \in X_{K}$ such that

Sequence $\left(x_{p q r}\right)$ is defined by $x_{p q r}=1$, for all $p, q, r \in N$

and sequence $\left(y_{p q r}\right)$ is defined by

$\left(y_{p q r}\right)=\left\{\begin{array}{l}\left(x_{p q r}\right), \text { when } p, q, \quad r \text { are even } \\ 0, \text { otherwise }\end{array}\right.$

Then $\left(x_{p q r}\right) \in c_{I}^{3}(F)$ but its $K$-step space pre-image does not belong to $c_{I}^{3}(F)$.

Therefore the sequence space $c_{I}^{3}(F)$ is not monotonic.

This complete the proof.

Theorem 3.6. The triple sequence spaces $c_{0 I}^{3}(F), c_{I}^{3}(F), l_{\infty I}^{3}(F), m_{I}^{3}(F)$ and $m_{0 I}^{3}(F)$ are sequence algebras.

Proof. To prove it we consider the sequence space $c_{0 I}^{3}(F)$, for the rest of the cases the proof will follow similarly.

Let $\left(x_{p q r}\right),\left(y_{p q r}\right) \in c_{0 I}^{3}(F)$

Then we have $I-\lim f_{p q r}\left(\left|x_{p q r}\right|\right)=0$, and $I-\lim f_{p q r}\left(\left|y_{p q r}\right|\right)=0$,

Now we obtain $I-\lim f_{p q r}\left(\left|x_{p q r} . y_{p q r}\right|\right)=0$,

It implies that $\left(x_{p q r} . y_{p q r}\right) \in c_{0 I}^{3}(F)$ is a sequence algebra

Hence the proof.

Theorem 3.7. The sequence spaces $c_{0 I}^{3}(F), c_{I}^{3}(F)$ and $l_{\infty I}^{3}(F)$ are not convergence free in general.

Proof. To prove it we consider a counter example for the space $c_{I}^{3}(F)$.

Let $I=I_{f}$ and $f(x)=x$, for all $x \in[0, \infty)$ 
Now we consider two triple sequence $\left(x_{p q r}\right)$ and $\left(y_{p q r}\right)$ such that

$\left(x_{p q r}\right)=\frac{1}{p+2 q+3 r}$, for all $p, q, r \in N$

and

$\left(y_{p q r}\right)=p+2 q+3 r$, for all $p, q, r \in N$

It can be easily verified that $\left(x_{p q r}\right) \in c_{I}^{3}(F)$ and $\left(y_{p q r}\right) \notin c_{I}^{3}(F)$

Hence the space $c_{I}^{3}(F)$ is not convergence free.

Similarly the others.

Theorem 3.8. If $I$ is neither maximal nor $I \neq I_{f}$ then the triple sequence spaces $c_{0 I}^{3}(F)$ and $c_{I}^{3}(F)$ are not symmetric in general.

Proof. Consider $A \in I$ be infinite and $f(x)=x$, for all $x \in[0, \infty)$.

If $\left(x_{p q r}\right)=\left\{\begin{array}{l}2, \quad \text { for all } p, \quad q, \quad r \in A \\ 0, \quad \text { otherwise }\end{array}\right.$

Then by Lemma 2.1 we can have $\left(x_{p q r}\right) \in c_{0 I}^{3}(F) \subset c_{I}^{3}(F)$.

Now we consider $K \subset N$ such that $K \notin I$ and $N-K \notin I$.

Let $\chi: K \rightarrow A$ and $\psi: N-K \rightarrow N-A$ be bijections, then the map $\pi: N \rightarrow N$ defined by

$\pi(p q r)=\left\{\begin{array}{l}\chi(p, q, r), \quad \text { for all } p, \quad q, \quad r \in K \\ \psi(p, q, r), \quad \text { otherwise }\end{array}\right.$

is a permutation on $N$, but $\left\{x_{(\pi(p, q, r))}\right\} \notin c_{0 I}^{3}(F)$ and $\left\{x_{(\pi(p, q, r))}\right\} \notin c_{I}^{3}(F)$.

Hence the sequence spaces $c_{0 I}^{3}(F)$ and $c_{I}^{3}(F)$ are not symmetric.

This complete the proof.

Acknowledgement. The author would like to thanks to the University Grants Commission North Eastern Regional Office, Guwahati, India for given the financial support (Ref: No. F.5-330/2015-16/MRP/NERO/1082). 


\section{References}

[1] A. J. Datta, A. Esi, B. C. Tripathy, Statistically convergent triple sequence spaces defined by Orlicz function, J. Math. Anal., 4(2), pp. 16-22, (2013).

[2] A. Sahiner, B. C. Tripathy, Some I-related properties of Triple sequences, Selcuk. J. Appl. Math., 9(2), pp. 9-18, (2008).

[3] A. Sahiner, M. Gurdal, K. Duden, Triple sequences and their statistical convergence, Selcuk. J. Appl. Math., 8(2), pp. 49-55, (2007).

[4] B. C. Tripathy, Statistically convergent double sequence, Tamkang. J. Math., 34(3), pp. 231-237, (2003).

[5] B. C. Tripathy, M. Sen, Paranormed I-convergent double sequence spaces associated with multiplier sequences, KYUNGPOOK Math. J. 54, pp. 321-332, (2014).

[6] B.C. Tripathy, R. Goswami, On triple difference sequences of real numbers in probabilistic normed spaces, Proyecciones J. Math., 33(2), pp. 157-174, (2014).

[7] B.C. Tripathy, R. Goswami, Vector valued multiple sequences defined by Orlicz functions, Bol. Soc. Paran. Mat., 33 (1), pp. 67-79, (2015).

[8] B.C. Tripathy, R. Goswami, Multiple sequences in probabilistic normed spaces, Afrika Matematika, 26 (5-6), pp. 753-760, (2015).

[9] B.C. Tripathy, R. Goswami, Fuzzy real valued p-absolutely summable multiple sequences in probabilistic normed spaces, Afrika Matematika, 26 (7-8), pp. 1281-1289, (year).

[10] E. Savas, R. F. Patterson, Double sequece spaces defined by a modulus, Math. Slovaca, 61, pp. 245-256, (2011).

[11] H. Fast, Surla convergence statistique, Colloq. Math., 2, pp. 241-244, (1951).

[12] H. Nakano, Concave modulars, J. Math. Soc. Japan, 5, pp. 29-49, (1953). 
[13] I. J. Schoenberg, The integrability of certain functions and related summability methods, Amer. Math. Monthly, 66, pp. 361-375, (1959).

[14] P. Kostyrko, T. Salat, W. Wilczynski, I-convergence, Real Anal. Exch. 26(2), pp. 669-686, (2000).

[15] S. Debnath, B. C. Das, Some new type of difference triple sequence spaces, Palestine J. Math.Vol. 4(2), pp. 284-290, (2015).

[16] S. Debnath, B. Sharma, B. C. Das, Some generalized triple sequence spaces of real numbers, J. Nonlinear Anal. Opti. 6(1) , pp. 71-79, (2015).

[17] S. Debnath, B. C. Das, D. Bhattacharya, J. Debnath, Regular matrix transformation on triple sequence spaces. Bol. Soc. Paran. Mat. 35(1), pp. 85-96, (2017)(In Press)

[18] T. Salat, B. C. Tripathy, M. Ziman, On some properties of Iconvergence, Tatra Mountain Mathematical Publications, pp. 669-686, (2000).

[19] V. Kumar, On I-convergence of double sequences Math. Commun., 12, pp. 171-181, (2007).

[20] V. A. Khan, N. Khan, On some I-convergent double sequence space defined by a modulus function, Scientific Research, 5, pp. 35-40, (2013).

[21] V. A. Khan, N. Khan, On some I-convergent double sequence spaces defined by a sequence of modulii, Ilirias Journal of Mathematics, 4(2), pp. 1-8, (2013).

\section{Bimal Chandra Das}

Department of Mathematics

Govt. Degree College,

Kamalpur - 799285

Tripura University

Dhalai, Tripura,

INDIA

e-mail : bcdas3744@gmail.com 